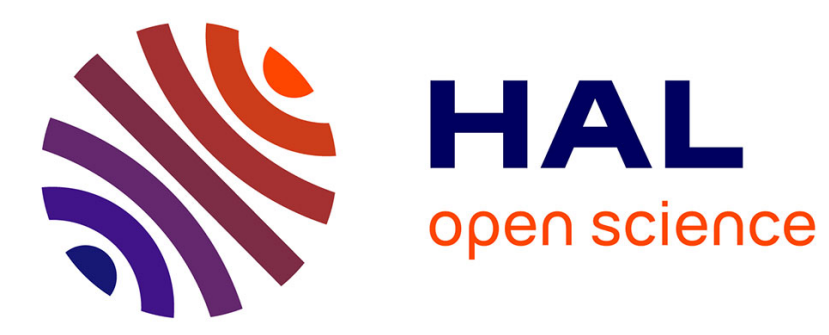

\title{
Oscillatory critical amplitudes in hierarchical models
}

Bernard Derrida, C. Itzykson, J. Luck

\section{To cite this version:}

Bernard Derrida, C. Itzykson, J. Luck. Oscillatory critical amplitudes in hierarchical models. Communications in Mathematical Physics, 1984, 94 (1), pp.115-132. 10.1007/BF01212352 . hal-03285925

\section{HAL Id: hal-03285925 \\ https://hal.science/hal-03285925}

Submitted on 19 Jul 2021

HAL is a multi-disciplinary open access archive for the deposit and dissemination of scientific research documents, whether they are published or not. The documents may come from teaching and research institutions in France or abroad, or from public or private research centers.
L'archive ouverte pluridisciplinaire HAL, est destinée au dépôt et à la diffusion de documents scientifiques de niveau recherche, publiés ou non, émanant des établissements d'enseignement et de recherche français ou étrangers, des laboratoires publics ou privés. 


\title{
Oscillatory Critical Amplitudes in Hierarchical Models
}

\author{
B. Derrida, C. Itzykson, and J. M. Luck
}

Service de Physique Théorique, CEN-Saclay, F-91191 Gif-sur-Yvette Cedex, France

\begin{abstract}
We study the oscillatory critical amplitudes of the $q$-states Potts model on a diamond hierarchical lattice. We consider an example of the generic case (finite critical index), as well as the degenerate case (essential singularity). In both cases, we compare the magnitude of the oscillations with geometrical characteristics of the Julia set of zeroes of the partition function.
\end{abstract}

\section{Introduction}

Much interest has been recently devoted to statistical mechanical models on hierarchical lattices $[1-10]$. On such lattices, the models can be solved by writing an exact renormalization group transformation. In particular one can compute exactly the free energy and all its derivatives. One can also know the whole set of singularities of this free energy in the complex temperature plane. It has been shown for many examples [7-10] that these singularities are located on the Julia set [10-16] associated with the renormalization transformation, i.e. the smallest closed set which contains all the unstable periods and their preimages. In the simplest cases [10], where one expects a single phase transition, the Julia set contains only a few points on the real axis. On the contrary, in some frustrated cases [5-9], one finds that the Julia set has an infinite number of points on the real axis, giving rise to an infinite number of critical temperatures [7].

Recently a relationship [17] between the shape of the density of zeroes, the critical exponents and the critical amplitudes has been proposed. This was a motivation for the study of analogous relationships in the case of hierarchical models. In this case, the critical exponent (which characterizes the singular behavior of the free energy at a critical point) can be easily extracted by linearizing the renormalization transformation at the fixed point. The shape of the Julia set can also be found easily [10]. As we shall see, the critical amplitudes are more difficult to obtain. It has been observed [7-10] in many cases that the critical behavior on hierarchical lattices is modulated: instead of observing as a leading singularity a pure power law, one finds a power law multiplied by a periodic function of the logarithm of the distance to the critical point.

The purpose of the present paper is to relate these oscillatory amplitudes to the shape of the Julia set near the critical point.

We shall consider successively two cases: the generic one, where the critical point is an isolated fixed point, and the degenerate one, where the critical point is a fixed point of multiplicity two. In both cases we shall determine numerically the shape of the Julia set and the oscillatory critical amplitudes. In the degenerate case, 
we shall have to consider a simplified model which allows for a numerical analysis. We shall give simple arguments allowing us to relate the order of magnitude of the oscillations to the shape of the Julia set near the fixed point.

\section{The Model}

In the following, we consider the Potts model on the diamond hierarchical lattice defined in [10]. The Hamiltonian of this model reads

$$
H=-J \sum_{\langle i j\rangle} \delta_{\sigma_{i} \sigma_{j}},
$$

where the $\sigma_{i}$ can take $q$ values $\left(\sigma_{i}=1,2, \ldots, q\right)$ and the sum runs over all nearestneighbor pairs. The recursion relations obeyed by the partition function lead to the following functional equation for the reduced free energy per bond:

$$
f[T(y)]=4 f(y)-2 \ln (2 y+q-2) .
$$

The renormalization mapping $T$ is

$$
y \mapsto T(y)=\left(\frac{y^{2}+q-1}{2 y+q-2}\right)^{2},
$$

where $y=\exp (\beta J)$. The domains $0 \leqq y \leqq 1$ and $y \geqq 1$ corresponds to the antiferromagnetic and ferromagnetic Potts models respectively.

For an arbitrary value of $q(q>0)$, the transformation $T$ has a fixed point $y_{c}>1$, corresponding to the critical temperature of the ferromagnetic model. The derivative of the map at its fixed point $\mu=T^{\prime}\left(y_{c}\right)$, is larger than one, since the temperature is a relevant parameter in the usual language of the renormalization group. In Sect. 3 we shall study the associated critical amplitudes for the value $q=2$ (Ising model), which is typical of the generic case.

When $q$ goes to zero, the fixed point $y_{c}$ goes to unity, $y_{c}=1+\sqrt{2 q}+\mathcal{O}(q)$. In other words, the critical point and the high temperature fixed point $(y=1)$ coalesce for $q \rightarrow 0$. This degenerate case is studied in Sect. 3 . In that case, the derivative of $T$ is $\mu=1$ : the fixed point is indifferent, or equivalently, temperature becomes marginal.

\section{The Generic Case $(q=2)$}

The transformation $T$ corresponding to the hierarchical Ising model $(q=2)$ has the simple form

$$
T(y)=\frac{1}{4}\left(y+\frac{1}{y}\right)^{2}
$$

It maps $y$ and $\frac{1}{y}$ onto the same point. This property, which is particular to $q=2$, leads to the following relation between the ferromagnetic and antiferromagnetic models:

$$
f(y)=f\left(\frac{1}{y}\right)+\ln y .
$$




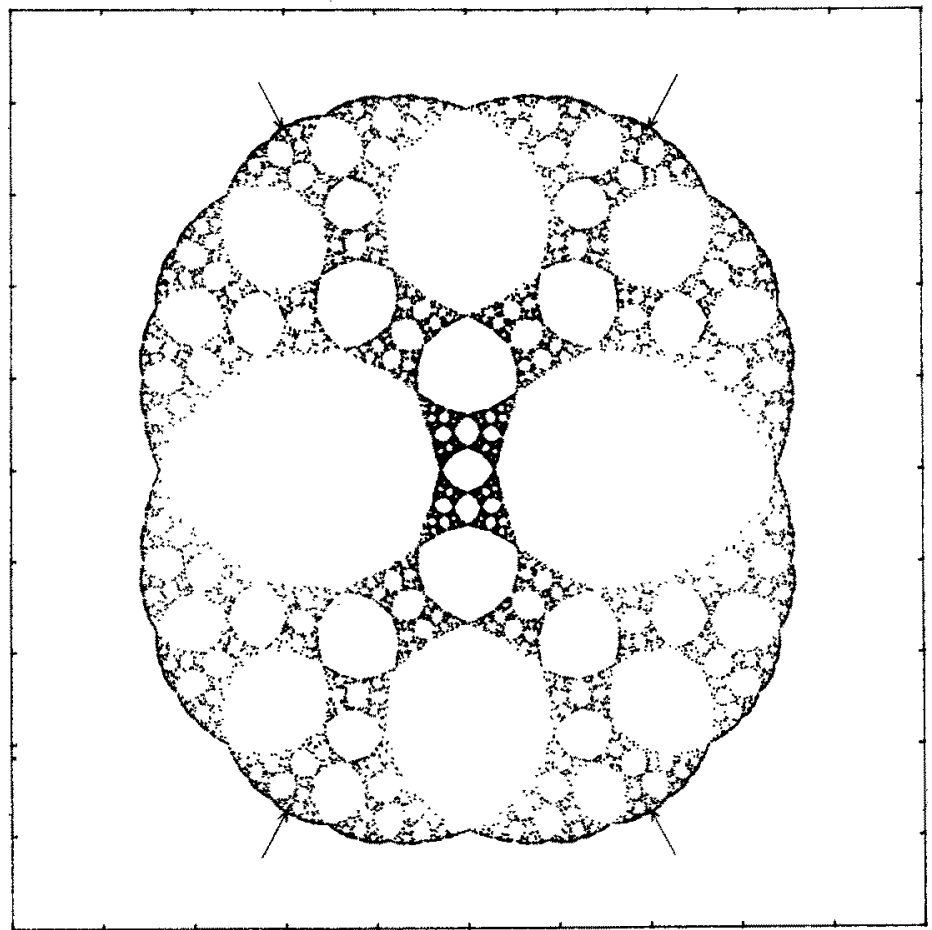

Fig. 1. The Julia set associated to the map of the generic case $(q=2)$. The arrows indicate the four extremal points of the set $S$ [see Eq.(29)]

We can therefore restrict our analysis to the vicinity of the ferromagnetic fixed point,

$$
y_{\varepsilon}=3.382975767 \ldots,
$$

where the derivative of $T$ reads

$$
\mu=1.678573510 \ldots
$$

The free energy $f$ admits the following convergent representation:

$$
f(y)=\frac{1}{2} \sum_{n \geqq 0} 4^{-n} \ln \left[2 T^{n}(y)\right],
$$

which is a consequence of Eq. (2).

The rest of this section is devoted to the properties of $f(y)$ around $y_{\varepsilon}$, and how they are related to the Julia set of the map $T$.

The Julia set associated with this map, shown in [10], is reproduced on Fig. 1.

\subsection{The Linear Approximation}

Let us first apply to our problem the method described in [18], which consists in linearizing the transformation $T$ around $y_{c}$, i.e. in replacing $T$ by the linear map $T^{(L)}$,

$$
T^{(L)}(y)=y_{c}+\mu\left(y-y_{c}\right)
$$


with

$$
\frac{y-y_{c}}{y_{c}}=x
$$

The prediction of the linear approximation $f^{(L)}$ of $f$ reads

$$
f^{(L)}(y)=\frac{2}{3} \ln \left(2 y_{c}\right)+\frac{1}{2} \sum_{n \geqq 0} 4^{-n} \ln \left(1+\mu^{n} x\right)
$$

and $f^{(L)}$ satisfies

$$
f^{(L)}\left[T^{(L)}(y)\right]-4 f^{(L)}(y)=-2 \ln (2 y) .
$$

The solutions of the homogeneous equation (obtained from (12) by setting the right-hand side equal to zero) read in the variable $x$

$$
F(x)=x^{a} P\left(\frac{\ln x}{\ln \mu}\right)
$$

where the critical index $a$ is

$$
a=\frac{\ln 4}{\ln \mu}=2.676531579 \ldots,
$$

and where $P$ is a periodic function, with period unity.

The same oscillatory behavior is expected for the singular part of $f(y)$, beyond the linear approximation. These oscillations of critical amplitudes are closely related to the discreteness of the renormalization transformation $[7,10,15,18]$.

In the linear approximation, the Fourier coefficients of the singular part of $f^{(L)}$ can be obtained in closed form, using the Mellin transformation.

Let $M(s)$ be the Mellin transformation of the sum appearing in Eq.(11),

$$
M(s)=\int_{0}^{\infty} x^{s-1} d x\left[\frac{1}{2} \sum_{n \geqq 0} 4^{-n} \ln \left(1+\mu^{n} x\right)\right] .
$$

The change of variable $x \rightarrow \mu^{n} x$ in each term allows us to perform the sum over $n$ explicitly,

$$
M(s)=\frac{1}{2} \frac{4 \mu^{s}}{4 \mu^{s}-1} \int_{0}^{\infty} x^{s-1} d x \ln (1+x) .
$$

This integral representation is convergent for $-1<\operatorname{Re} s<0$, where we have

$$
M(s)=\frac{1}{2} \frac{4 \mu^{s}}{4 \mu^{s}-1} \frac{\pi}{s \sin (\pi s)} .
$$

The analytic continuation of $M(s)$ has poles at $s=-n(n=1,2,3, \ldots)$ and at $s=-(a+i p \omega)(p=0, \pm 1, \pm 2, \ldots)$, where $a$ is defined in Eq. (14), and where

$$
\omega=\frac{2 \pi}{\ln \mu} .
$$

The contributions of these two series of poles give rise to a regular (reg) part and a singular (sg) part of $f^{(L)}$, respectively, for $x \rightarrow 0$. 
We have therefore

$$
f^{(L)}(y)=f_{\mathrm{reg}}^{(L)}(y)+f_{\mathrm{sg}}^{(L)}(y)
$$

where

$$
f_{\mathrm{reg}}^{(L)}(y)=\frac{2}{3} \ln \left(2 y_{c}\right)+\frac{1}{2} \sum_{n \geqq 0} \frac{(-)^{n-1}}{n} \frac{x^{n}}{1-\frac{\mu^{n}}{4}}
$$

is analytic in the disk $|x|<\mu$; and where

$$
f_{\mathrm{sg}}^{(L)}(y)=x^{a} \sum_{-\infty<p<+\infty} A_{p}^{(L)} e^{i p \omega \ln x}
$$

is a periodic function of $\frac{\ln x}{\ln \mu}$ with the following Fourier coefficients:

$$
A_{p}^{(L)}=\frac{\pi}{2 \ln \mu(a+i p \omega) \cdot \sin [\pi(a+i p \omega)]} .
$$

In order to observe the oscillatory behavior of $f(y)$, let us consider its third derivative

$$
B(y)=\frac{d^{3} f(y)}{d y^{3}}
$$

$B$ is expected to exhibit the following divergent behavior (since $a-3$ is negative):

$$
B(y) \sim\left(y-y_{c}\right)^{a-3} \psi(y) .
$$

The linear approximation $\psi^{(L)}(y)$ to the "critical amplitude" $\psi(y)$ is an oscillatory function with very rapidly decreasing Fourier coefficients. All harmonics except the first one are therefore very hard to measure.

Define the average value $\psi_{\mathrm{av}}$ and the relative oscillation amplitude $\eta$ by:

$$
\begin{gathered}
\psi_{\mathrm{av}}=\frac{1}{2}\left[\psi_{\max }+\psi_{\min }\right], \\
\eta=\frac{\psi_{\mathrm{max}}-\psi_{\min }}{\psi_{\mathrm{av}}} .
\end{gathered}
$$

The predictions of the linear approximation for these quantities can be easily derived from Eqs. (19) and (20). If we take only $A_{0}, A_{ \pm 1}$ into account, we get:

$$
\begin{gathered}
\psi_{\mathrm{av}}^{(L)}=\frac{(a-1)(a-2)}{2 y_{c}^{a} \ln \mu} \frac{\pi}{\sin (\pi a)}, \\
\eta^{(L)}=\frac{4 \sin (\pi a)}{(a-1)(a-2)}\left|\frac{(a-1+i \omega)(a-2+i \omega)}{\sin [\pi(a+i \omega)]}\right|,
\end{gathered}
$$

which gives numerically

$$
\psi_{\mathrm{av}}^{(L)}=0.15501477 \ldots, \quad \eta^{(L)}=2.507195 \ldots 10^{-14} .
$$




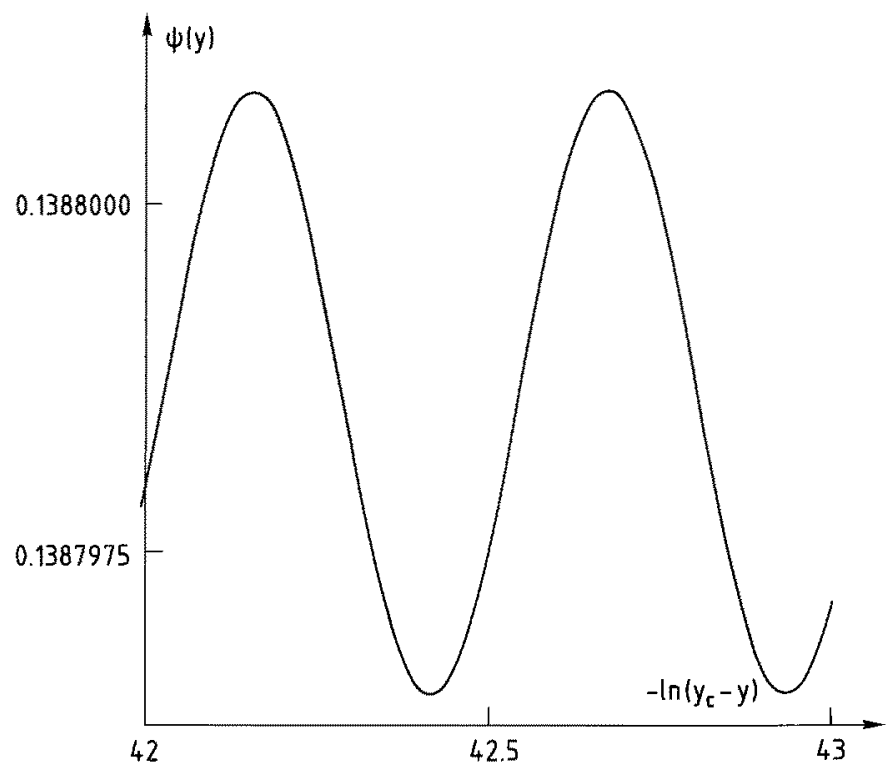

Fig. 2. The critical amplitude $\psi$ of the third derivative of the free energy near the critical point in the high-temperature phase $\left(y<y_{\mathrm{c}}\right)$ [see Eq. (22)]

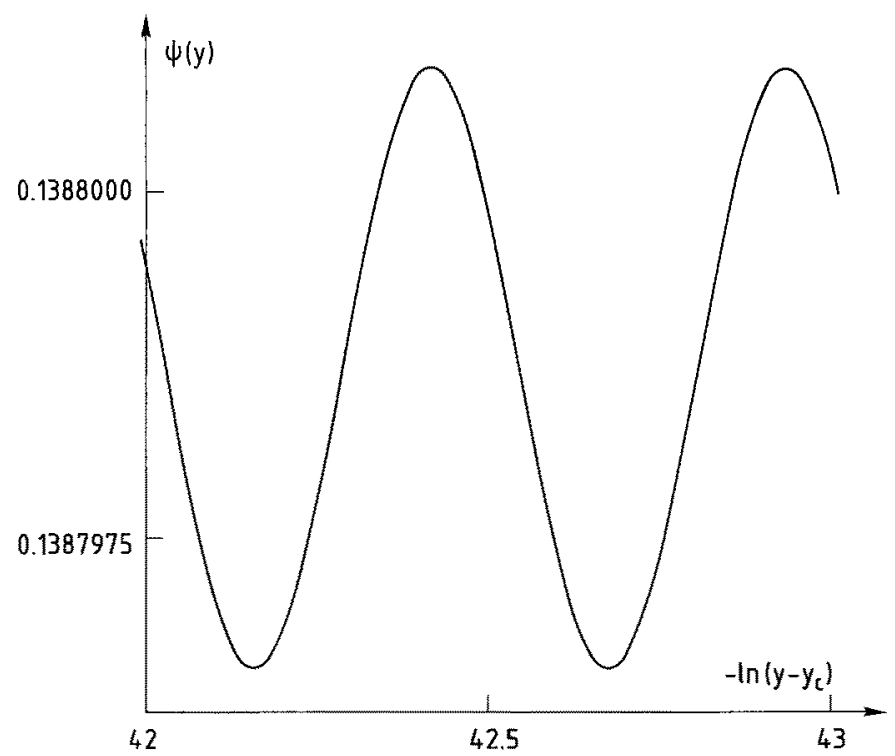

Fig. 3. The same as in Fig. 2, for the low-temperature phase $\left(y>y_{c}\right)$

\subsection{Numerical Results}

The expression given in Eq. (8) for $f(y)$ leads easily to a series representation for its third derivative $B(y)$.

We have treated numerically this series representation of $B(y)$, for $y$ very close to $y_{c}$. In order to isolate the leading critical behavior, we had to choose $\left(y-y_{c}\right)$ of the order of $10^{-19}$, and to use double precision on a CRAY. The results for the amplitude $\psi(y)$ defined in Eq. (22) are represented on Figs. 2 and 3, for $y<y_{c}$ and $y>y_{c}$, respectively. 
These figures show clearly that $\psi(y)$ is asymptotically a periodic function of $\ln \left(\left|y-y_{c}\right|\right)$ with period $\ln \mu$, and that higher harmonics are very small.

Both phases $\left(y>y_{c}\right.$ and $\left.y<y_{c}\right)$ lead to the same values for $\psi_{\mathrm{ar}}$ and $\eta$ (after extrapolation over a wider range than the one represented on Figs. 2 and 3), namely:

$$
\psi_{\mathrm{av}}=0.13879868 \ldots, \quad \eta=3.1248 \ldots 10^{-5} .
$$

As far as $\psi_{\text {av }}$ is concerned, the prediction (25) of the linear theory gives a satisfactory estimate, with $11 \%$ relative accuracy. On the contrary, the relative amplitude of oscillations $\eta$ is 9 orders of magnitude bigger than the estimate of the linear approximation. The next subsection is devoted to the explanation of such a large discrepancy.

\subsection{Oscillations and Angles}

The crucial property missed by the linear approximation is that $f(y)$ is analytic only in a sector: $\left|\arg \left(y-y_{c}\right)\right|<\theta_{1}$, as can be seen on Fig. 1 .

The function $f^{(L)}(y)$ defined by (11) is analytic in a cut plane $\left(\left|\arg \left(y-y_{c}\right)\right|<\pi\right)$, since the large order behavior of the $A_{p}^{(L)}$ reads

$$
A_{p}^{(L)} \sim \exp (-\pi \omega|p|) \text {. }
$$

On the other hand, if $\theta_{1}$ is the limiting angle of analyticity of $f$, the Fourier coefficients $A_{p}$ of $f$ for $y>y_{c}$ decrease only as rapidly as

$$
A_{p} \sim \exp \left(-\omega \theta_{1}|p|\right) \text {. }
$$

Let us now try to determine the angle $\theta_{1}$ appearing in Eq. (28). Fig. 4 represents an enlargement of the Julia set around the fixed point $y_{r}$.

This picture suggests that the Julia set lies outside an extremal angle $\tilde{\theta}_{1}$, and $f(y)$ is certainly analytic in the sector $\theta<\widetilde{\theta}_{1}$, and therefore $\theta_{1} \geqq \widetilde{\theta}_{1}$. In the following, we shall conjecture that $\theta_{1}$ and $\widetilde{\theta}_{1}$ are equal.

From Fig. 4, one gets a rather poor estimate of the angle $\theta_{1}$. One way to get a better accuracy is to generate by a biased Monte Carlo method [10] a large sample of points of the Julia set, and to find the smallest angle defined by this sample. The resulting measurement systematically gives a larger $\theta_{1}$ than the one obtained using the following procedure.

Consider the set $S$ given by

$$
\begin{gathered}
\qquad \begin{array}{c}
S=\left\{y_{1}, \bar{y}_{1},-y_{1},-\bar{y}_{1}\right\}, \\
\text { where } y_{1} \text { satisfies } T\left(y_{1}\right)=\bar{y}_{1},
\end{array} \\
\text { and therefore } y_{1}=-2.006162159 \ldots+3.744147122 \ldots i .
\end{gathered}
$$

Clearly these points belong to the Julia set, because of Eq. (30). This set leads to the following estimate of $\theta_{1}$ :

$$
\theta_{1}=\lim _{n \rightarrow \infty} \operatorname{Arg}\left[\left(T^{-1}\right)^{n}\left(y_{1}\right)-y_{c}\right],
$$

where $T^{-1}$ stands for the one among the 4 branches of $T^{-1}$ which ensures convergence towards $y_{c}$. The numerical value obtained in this way reads

$$
\theta_{1}=1.159171214 \ldots .
$$




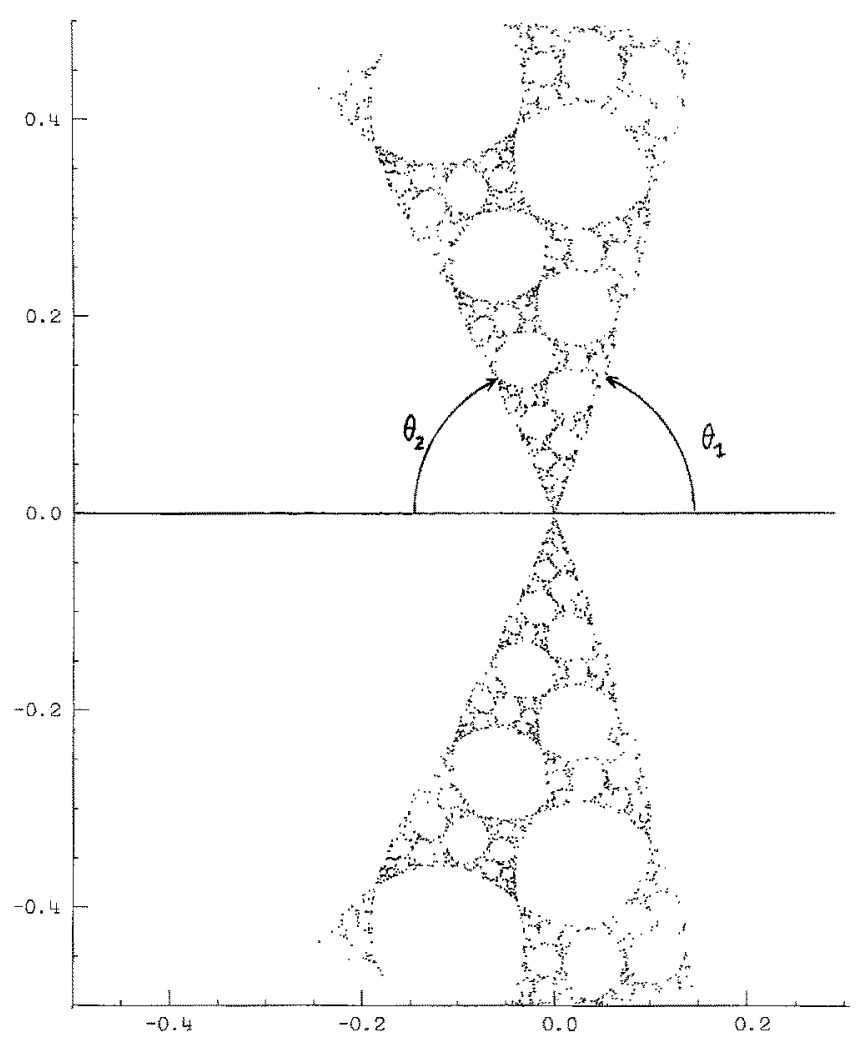

Fig. 4. An enlargement of the Julia set of the case $q=2$ around the ferromagnetic fixed point $y_{c}$, showing the limiting angles $\theta_{1}, \theta_{2}$

A heuristic justification for our choice (29) for the set $S$ is as follows. Consider the extremal points $y_{c}$, defined as being those of the Julia set having the largest modulus. These points are clearly the most "exterior" ones, and those of their preimages which converge towards $y_{c}$ are also locally the most "exterior" ones. As a consequence, an estimate (upper bound) on $\theta_{1}$ can be extracted from these points.

Moreover, the points of $S$ can be characterized in another way. Let $\mu_{n}$ be the moments of the invariant balanced measure on the Julia set (see [15] for the properties of $d \mu$ ):

$$
\mu_{n}=\int_{J} y^{n} d \mu(y), \quad-\infty \leqq n \leqq+\infty .
$$

For large positive $n, \mu_{n}$ is asymptotically dominated by the points of largest modulus. On the other hand, since $d \mu$ is invariant under $T$, the $\mu_{n}$ satisfy a recursion relation. If we assume that this relation is also fulfilled by the asymptotic behavior of the $\mu_{n}$, one gets precisely the set $S$ defined by Eqs. (28) and (29).

By inspection of Fig. 1, these four points (indicated by arrows) are very likely to be the extremal points of the Julia set. However, our derivation of $S$ need not work in other circumstances, as can be verified by studying the moments $\left\langle(y-a)^{n}\right\rangle$ with a variable origin $a$. 
This approach has another interesting feature. The equation $T(y)=\bar{y}$ has actually four solutions: $y_{1}, \bar{y}_{1}$ and $y_{2}, \bar{y}_{2}$ with:

$$
y_{2}=0.440964442 \ldots+0.4801893411 \ldots i \text {. }
$$

The angle $\theta_{2}$ associated to $y_{2}$ through Eq. (32) happens to be (numerically)

$$
\theta_{2}=-\theta_{1} \text {. }
$$

Figure 4 seems to indicate that this angle $\theta_{2}$ coincides with the internal angle of the Julia set at $y_{c}$.

We can now formulate an improved estimate for the relative oscillation amplitude $\eta$. From Eqs. (27-28), we have the following asymptotic relation between the genuine Fourier coefficients $A_{p}$ and their approximations $A_{p}^{(L)}$ from the linear theory:

$$
\frac{A_{p}}{A_{p-1}} \sim\left(\frac{A_{p}^{(L)}}{A_{p-1}^{(L)}}\right)^{\theta_{1} / \pi} .
$$

Since this number is very small, the $A_{p}$ are very rapidly decreasing, and we are tempted to extend the validity of Eq. (37) down to $p=1$.

This leads to

$$
\eta \sim 4\left(\frac{\eta^{(L)}}{4}\right)^{\theta_{1} / \pi}=229903 \ldots 10^{-5} .
$$

This value is now only $26 \%$ below the exact value given in Eq. (26).

Moreover, the fact that the amplitudes $\psi_{\mathrm{av}}$ and $\eta$ are identical in both phases strongly suggests that all the $A_{p}$ 's are identical, up to $p=\infty$, and therefore, by using Eq. (28) and an analogous equation for $y<y_{c}$ using $\theta_{2}$, we deduce that the equality of the two angles limiting the Julia set around the point $y_{c}$ is very likely to be true (see note added in proof).

We have also generated a very large sample of points on the boundary of the Julia set (Fig. 5) close to the critical point. This picture as compared to Fig. 4 illustrates the fact that, both from a geometrical point of view as well as from a measure theoretical one, the oscillations in the free energy presumably reflect similar oscillations present in the Julia set. This suggests that the estimate (37), which is obviously true but only depends on a limiting angle, does not exhaust the question of a true evaluation of the first few oscillatory amplitudes $A_{p}$. Therefore the fact that our bold extrapolation gave such reasonable results is presumably merely to be considered as a lucky circumstance which might not occur in other cases.

\section{The Degenerate Case $(q \rightarrow 0)$}

As explained in Sect. 2, the case $q \rightarrow 0$ is particular, because the map $T$ has a derivative equal to unity at its fixed point. Therefore a new analysis is required.

Let us define the variable

$$
u=\frac{1}{4}(y-1)
$$

In terms of $u$, the map $T$ reads

$$
T(u)=u+u^{2},
$$




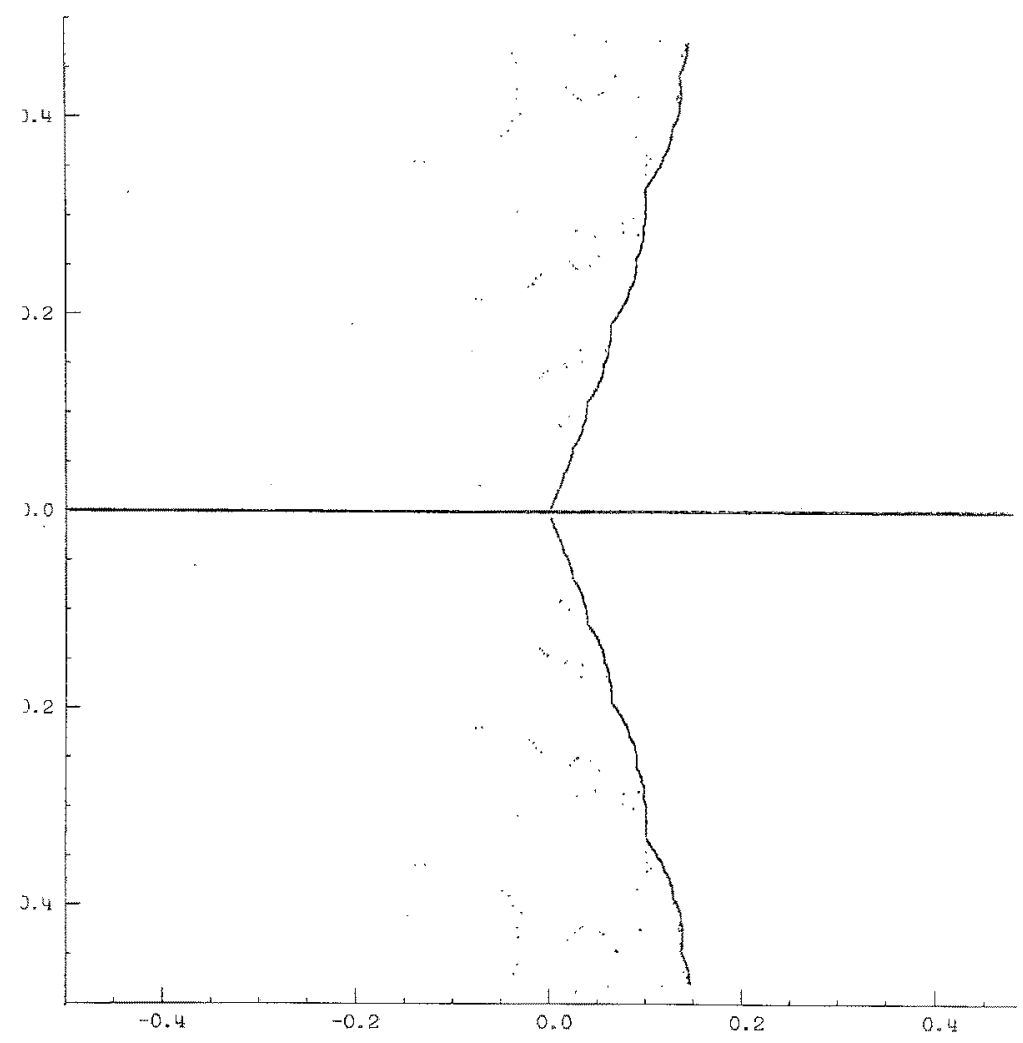

Fig. 5. An enlargement of the Julia set of the case $q=2$ around the ferromagnetic fixed point $y_{c}$, showing the oscillatory structure of the boundary of the set

and the renormalization group equation for the free energy is

$$
f\left(u+u^{2}\right)=4 f(u)-2 \ln 8 u .
$$

The free energy admits therefore the convergent representation:

$$
f(u)=\frac{1}{2} \sum_{n \geqq 0} 4^{-n} \ln \left[8 T^{n}(u)\right] .
$$

The Julia set associated to the map $T$ is represented on Fig. 6 . It was proved by Julia [11] to be a continuous curve; it seems nevertheless to have some structure at every length scale, just as in the generic case.

This section is devoted to the properties of the function $f(u)$, and to their relationship to the Julia set of the associated map. We shall focus upon the analogies and differences between the generic case and the degenerate one.

\subsection{The Continuous Approximation}

Let us consider the homogeneous equation associated to Eq. (41):

$$
\varphi\left(u+u^{2}\right)=4 \varphi(u)
$$




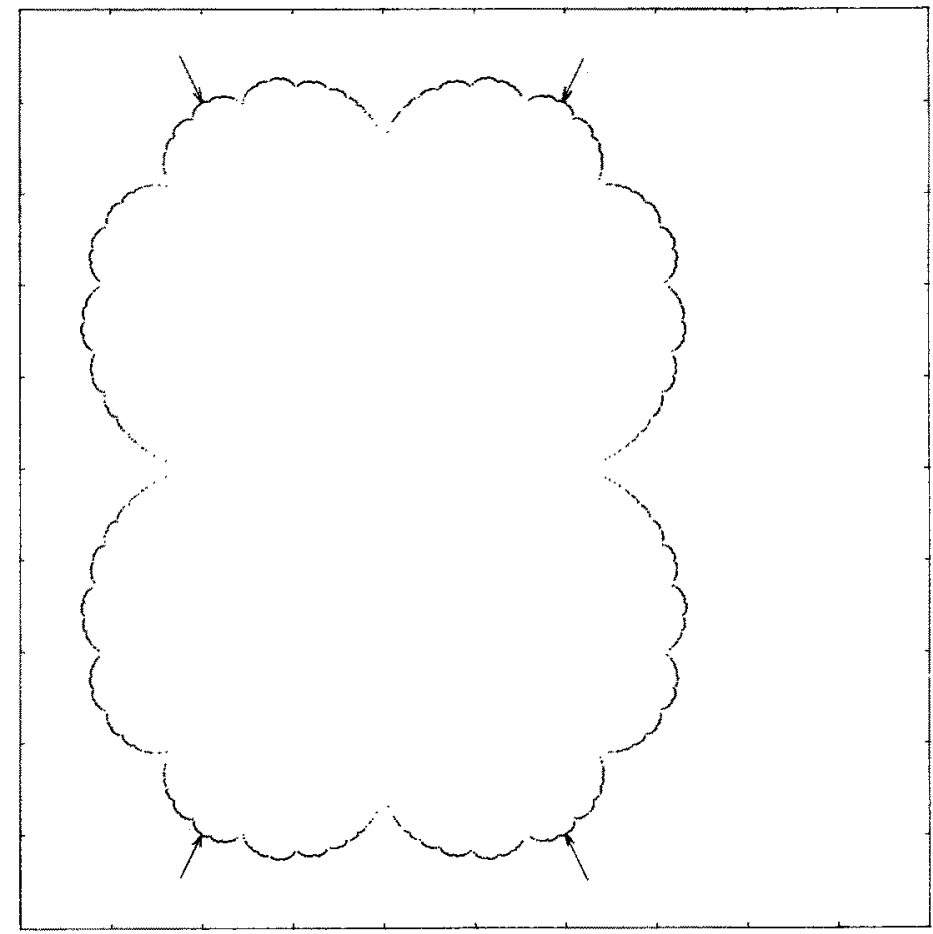

Fig. 6. The Julia set associated to the map: $T(u)=u+u^{2}$, corresponding to the degenerate case $(q \rightarrow 0)$. The arrows indicate the four extremal points of the set $S$ [see Eq. (74)]

We will show that the solutions of Eq. (43) exhibit an essential singularity at $u=0$, modulated by an asymptotically periodic function of $\left(\frac{1}{u}-\ln u\right)$. Define

$$
\gamma(u)=\ln \varphi(u)
$$

It follows from Eq. (43) that $\gamma$ satisfies

$$
\gamma\left(u+u^{2}\right)=\gamma(u)+\ln 4
$$

Assuming that $\gamma$ is differentiable for $u>0$, we deduce from Eq. (45) that, in the $u \rightarrow 0$ limit, we have

$$
\gamma^{\prime}(u) \sim \frac{\ln 4}{u^{2}}
$$

and we get the following very crude estimate:

$$
\varphi(u) \sim e^{-(\ln 4) / u} .
$$

This analysis can be pursued by keeping systematically higher order terms in the differential equation (46), assuming that the function $g(u)$ defined by

$$
\gamma^{\prime}(u)=\frac{\ln 4}{u^{2}} g(u)
$$

is regular around $u=0$. 
Under this assumption, we obtain

$$
g(u)=1+u-\frac{1}{2} u^{2}+\frac{2}{3} u^{3}-\frac{13}{12} u^{4}+\ldots .
$$

By inserting this expansion into Eqs. (44) (48), one gets the following "continuous approximation" $\varphi^{(c)}$ to the function $\varphi$, general solution of the homogeneous equation (43)

$$
\varphi^{(c)}(u)=e^{-(\ln 4) / u} u^{\ln 4}\left[1-\frac{\ln 4}{2} u+A_{2} u^{2}+\ldots\right] .
$$

Consider now the ratio $R_{\varphi}(u)$ between the function $\varphi$ and its continuous approximation

$$
R_{\varphi}(u)=\frac{\varphi(u)}{\varphi^{(c)}(u)}
$$

This "amplitude" satisfies

$$
R_{\varphi}(u)=R_{\varphi}\left(u+u^{2}\right) .
$$

This equation allows $R_{\varphi}$ to be a periodic function, with period unity, of a variable $x$ satisfying

$$
x\left(u+u^{2}\right)=x(u)-1 .
$$

Since Eq. (53) is, up to a multiplicative factor, identical to Eq. (45), we deduce from our previous study that $x(u)$ has the following behavior:

$$
x(u)=\frac{1}{u}-\ln u+x_{0}+\ldots .
$$

The continuous approximation predicts therefore that the general solution of Eq. (43) reads:

$$
\varphi(u)=e^{-(\ln 4) / u} u^{\ln 4} A(u) R_{\varphi}(u)
$$

where

$$
A(u)=1-\frac{\ln 4}{2} u+A_{2} u^{2}+\ldots
$$

is a universal function ( $A$ does not depend on the particular solution $\varphi$, and the $A_{n}$ are systematically computable), and $R_{\varphi}(u)$ is a periodic function in the variable $x$, which is asymptotic to $1 / u$.

The free energy $f(u)$ is also expected to have a singular part given by Eq. (55), by analogy with the generic case.

\subsection{A Toy-Model}

In the generic case, the oscillations modulate a power-law singularity, and it is sufficient to consider a given derivative of the free energy (the third one in the case $q=2$ ) to observe these oscillations numerically. 
In the present case, in order to extract the oscillatory behavior (55) of $f(u)$ from its smooth background, we should formally differentiate our function an infinite number of times.

We have nevertheless a more direct way of checking that the function $f(u)$ has indeed an essential singularity, as predicted by the continuous approximation. Unfortunately this method does not allow us to observe the oscillations.

If we insert into Eq. (41) the following expansion of $f$ :

$$
f(u)=f_{-1} \ln u+\sum_{n \geqq 0} f_{n} u^{n},
$$

we can compute the $f_{n}$ systematically

$$
\begin{gathered}
f_{-1}=\frac{2}{3} ; \quad f_{0}=\frac{2}{3} \ln 8 ; \quad f_{1}=\frac{2}{9} ; \\
f_{n}=\frac{1}{3}\left[\frac{2}{3} \frac{(-1)^{n-1}}{n}+\sum_{l}\left(\begin{array}{c}
l \\
n-l
\end{array}\right) f_{l}\right] \text { for } n \geqq 2,
\end{gathered}
$$

where $l$ runs from $\left[\left(\frac{n}{2}\right)\right]\left(\right.$ integer part of $\left.\frac{n}{2}\right)$ up to $n$ in the summation.

In order to explore the singularities of $f$, let us define its Borel transform

$$
B(t)=\sum_{n \geqq 0} B_{n} t^{n}, \quad \text { with } \quad B_{n}=\frac{f_{n}}{n !} .
$$

A singularity of the type given by (55) in $f$ implies that $B(t)$ has a power-law singularity at $t^{*}=\ln 4$,

$$
B(t) \sim K_{1}\left(t^{*}-t\right)^{\ln 4},
$$

and that the $B_{n}$ have the asymptotic behavior

$$
B_{n} \sim K_{2}(\ln 4)^{-n} n^{-(1+\ln 4)} .
$$

We present in Fig. 7 the result of a numerical determination of the $B_{n}$ (up to $n=400$ ). We plot the quantity

$$
y_{n}=\frac{1}{n} \ln B_{n}+\ln (\ln 4)
$$

vs. $(\ln n) / n$. The continuous line has a slope

$$
s=-(1+\ln 4) \text {. }
$$

It is therefore very clear that the Borel transform of $f(u)$ has a singularity at $t^{*}$, with an exponent $s$ which is correctly predicted by the continuous approximation.

This method is not accurate enough to predict that our function $f(u)$ has oscillations, i.e. that its Borel transform has also singularities at

$$
t_{k}^{*}=\ln 4+2 \pi i k, \quad k \neq 0 .
$$

In order to pursue the analysis of the present case and to study the relation between the oscillations of critical amplitudes and the shape of the Julia set, we choose to study in the remainder of this section a very similar function $F(u)$, 


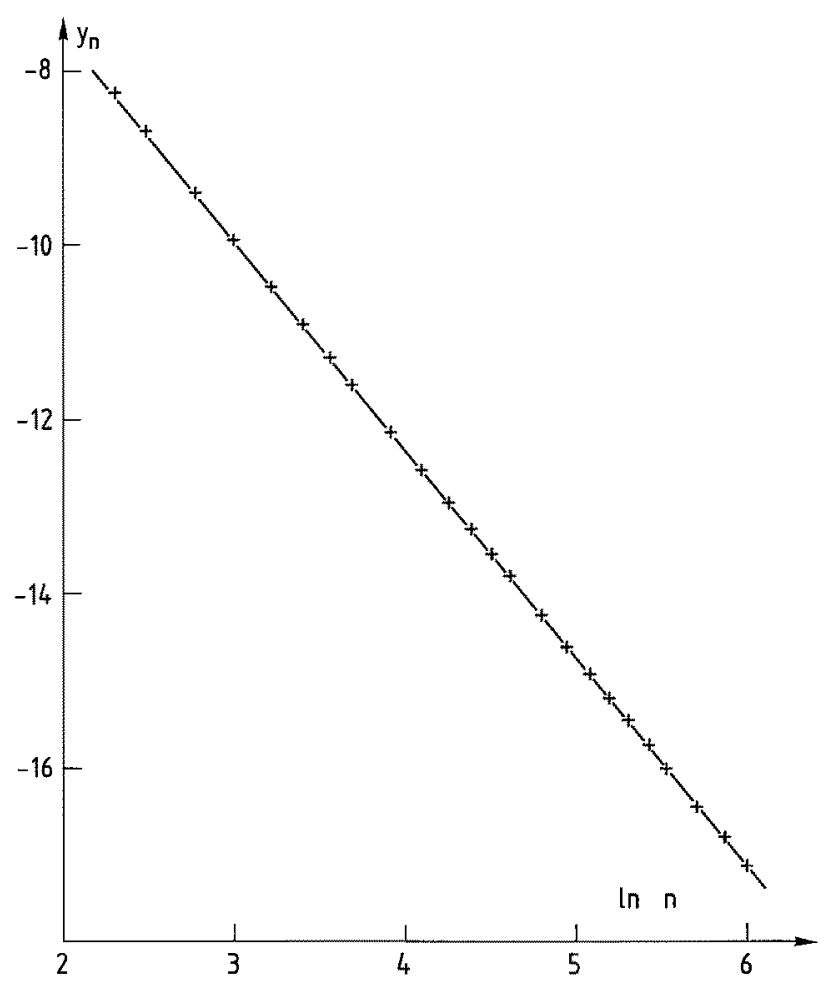

Fig. 7. The quantities $y_{n}$ [see Eq. (62)], related to the Taylor coefficients of the Borel transform of the free energy, in the degenerate case. The crosses are the numerical values; the continuous line has a slope $s=-(1+\ln 4)$

defined as being the logarithmic transform of the unique balanced measure $d \mu$ on the Julia set of the map $T$ (see [15] for a precise definition of $d \mu$ and its properties).

The function $F(u)$ satisfies

$$
\begin{gathered}
F\left(u+u^{2}\right)=2 F(u), \\
F(u) \sim \ln u \quad(u \rightarrow \infty) .
\end{gathered}
$$

In other physical instances, it might occur that a similar essential singularity appears in the free energy, but that its amplitude can hardly be measured, due to the presence of a regular background. Nevertheless other quantities (such as correlation lengths) might exhibit a pure singular behavior in one of the phases. Our model for $F(u)$ is designed to capture the structure of such quantities. Indeed, would one be tempted to define a correlation length on the diamond hierarchical lattice, its reciprocal $\xi^{-1}(u)$ would satisfy relation (64), but not necessarily the boundary condition (65).

Since Eq. (65) is homogeneous, our continuous approximation predicts the following behavior of $F$ around $u=0$ outside the Julia set,

$$
F(u)=e^{-(\ln 2) / u} u^{\ln 2}\left[1-\frac{\ln 2}{u}+F_{2} u^{2}+\ldots\right] R_{F}(u) .
$$


$F$ has the advantage of exhibiting a pure essential singularity and no smooth background. The oscillatory behavior of $R_{F}$ is therefore numerically observable. The easiest way to measure these oscillations is to consider the quantity

$$
E(u)=u \frac{d F}{d u} .
$$

It admits the following infinite product representation:

$$
E(u)=\prod_{n \geqq 0} \frac{\frac{1}{2}+T^{n}(u)}{1+T^{n}(u)},
$$

which converges for every $u$ outside the Julia set, i.e. such that $T^{n}(u) \rightarrow \infty$ when $n$ $\rightarrow \infty$.

The continuous approximation predicts for $E$ the following behavior:

$$
E(u)=e^{-(\ln 2) / u} u^{\ln 2-1}\left[1+E_{1} u+E_{2} u^{2}+E_{3} u^{3}+\ldots\right] R_{E}(u) .
$$

The few first $E_{n}$ 's read

$$
\begin{aligned}
& E_{1}=1-\frac{1}{2} \ln 2, \\
& E_{2}=-\frac{1}{2}-\frac{1}{6} \ln 2+\frac{1}{8}(\ln 2)^{2}, \\
& E_{3}=\frac{2}{3}+\frac{2}{9} \ln 2-\frac{1}{24}(\ln 2)^{2}-\frac{1}{48}(\ln 2)^{3} .
\end{aligned}
$$

\subsection{Numerical Results}

We have evaluated the function $E(u)$ through its infinite product representation (68), and extracted the amplitude $R_{E}$ from these data according to Eqs. (69) and (70), keeping only the first three coefficients $E_{n}$ 's.

Figure 8 shows a plot of the function $R_{E}(u)$ versus $\frac{1}{u}$ in the interval $[1000 ; 1002]$. $R_{E}$ is clearly very close to being a periodic function of $\frac{1}{u}$. Indeed the difference between $\frac{1}{u}$ and the variable $x(u)$ defined in Eqs. $(53-54)$ is not observable on a few periods range.

We can extract from these numerical data the average $\left(R_{E}\right)_{a v}$ and the relative oscillation amplitude $\eta$ of $R_{E}$, defined as in Sect. 3. These numbers read

$$
\left(R_{E}\right)_{\mathrm{av}}=0.81624968 \ldots, \quad \eta=8.1497 \ldots 10^{-7} .
$$

The main difference between the present degenerate case and the generic one is that we do not know of any a priori estimate of these numbers, like in the generic case. In next section we present a relationship between the oscillations of $R_{F}$ and $R_{E}$ and a geometrical characteristic of the Julia set.

\subsection{Oscillations and Radius of Curvature}

In the generic case, the Fourier coefficients of the amplitude of $f(y)$ were asymptotically governed by the angle $\theta_{1}$ of the domain of analyticity of $f$. 


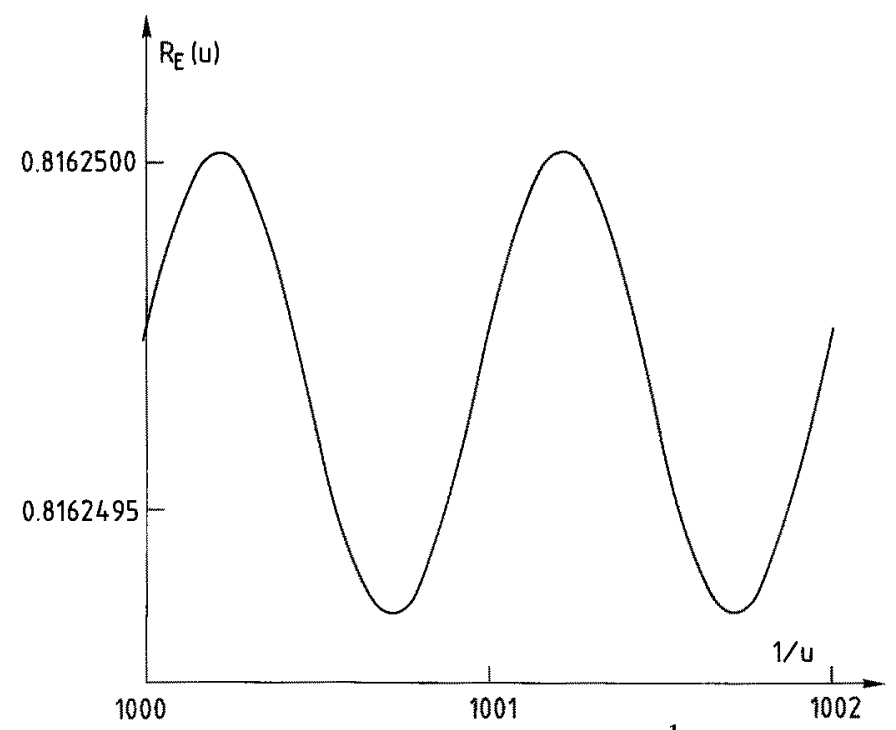

Fig. 8. The amplitude $R_{E}(u)$ of the toy-model function $E(u)$, vs. $\frac{1}{u}$ [see Eq. (51)]

In the present case, the function $F(u)$ is analytic in the exterior of the Julia set represented in Fig. 5. Let us assume that this set has a finite limiting radius of curvature $\varrho$ at the origin. Then the functions $F, E, R_{F}, R_{E}$ are asymptotically analytic functions of $\frac{1}{u}$ in the band

$$
\left|\operatorname{Im} \frac{1}{u}\right|<\frac{1}{2 \varrho}
$$

and the Fourier coefficients $\tilde{R}_{F}(n)$ and $\tilde{R}_{E}(n)$ of the functions $R_{F}$ and $R_{E}$ in the variable $\frac{1}{u}$ decreases as

$$
\widetilde{R}_{F}(n) \sim \widetilde{R}_{E}(n) \sim e^{-\pi|n| / e} .
$$

Let us now give a determination of $\varrho$, based on the concept of extremal points introduced in Subsect. 3.2. The set of extremal points reads in the present case

$$
S=\{+i,-i ;-1+i ;-1-i\},
$$

and therefore, if the radius $\varrho$ is determined by the preimages of $S$, we have

$$
\frac{1}{2 \varrho}=\lim _{n \rightarrow \infty} \frac{\operatorname{Im} z_{n}}{\left(\operatorname{Re} z_{n}\right)^{2}},
$$

where $z_{n}=\left(T^{-1}\right)^{n}(i)$ and $T^{-1}(y)=\sqrt{\frac{1}{4}+y}-\frac{1}{2}$.

These formulae lead to the following numerical results:

$$
\frac{1}{2 \varrho}=2.2156589 \ldots, \quad e^{-\pi / \varrho}=8.995279 \ldots 10^{-7}
$$


Since this last number is very small, the asymptotic estimate (73) should not be violently wrong for small $n$. Indeed, if we boldly extrapolate (73) down to $n=1$ with the same precautions as in Sect. 3, we obtain

$$
\eta=4 e^{-\pi / e}=3.5981 \ldots 10^{-6} \text {. }
$$

Compared to the measured value given by Eq. (71), this estimate is off by a factor of 4 , while the corresponding relative error was only of $26 \%$ in the generic case. This illustrates the point, made at the end of Sect. 3 , that the extrapolation of (73) down to $n=1$ gives only a rough estimate of $\eta$. This approach has nevertheless the advantage to let us understand how the existence of a finite least upper bound for the radius of curvature $g$ can explain a crucial property of the function $F$, namely the existence of oscillations in the variable $\frac{1}{u}$, and to predict correctly at least the order of magnitude of these oscillations.

\section{Conclusion}

We have shown how the shape of the Julia set near a critical point is related to the oscillations of the critical amplitudes. There is no doubt that these oscillations are produced by the discrete character of the renormalization transformation. Similar oscillations are of course present near the preimages of the fixed point, as well as near the preimages of critical periods [7].

The most important question is to know if these oscillations are actually present for models on Bravais lattices. Of course, any approximate real space renormalization $[18,19]$ on Bravais lattices will produce oscillatory amplitudes. It is reasonable to think that for simple phase transitions like the one of the ferromagnetic Ising model, these oscillations are not present, because the change of scale can become infinitesimal in the scaling limit. However, it has been shown recently that, in some one-dimensional models $[20,21]$, these oscillations do exist. Therefore they should not be considered always as parasitic effects of real space renormalizations.

Our study does not exhaust other interesting problems related to Julia sets [16], like the average escape rate or the Hausdorf dimension.

Acknowledgements. It is a pleasure to thank D. Bessis, L. De Seze, H. Flyvbjerg, J. Geronimo, and P. Moussa for fruitful discussions.

\section{References}

1. Berker, A.N., Ostlund, S.: Renormalization-group calculations of finite systems: order parameter and specific heat of epitaxial ordering. J. Phys. C12, 4961 (1979)

2. Kaufman, M., Griffiths, R.B.: Exactly soluble Ising models on hierarchical lattices. Phys. Rev. B24, 496 (1981)

3. Kaufman, K., Griffiths, R.B.: Infinite susceptibility at high temperature in the MigdalKadanoff scheme. I. Phys. A15, L239 (1982)

4. Griffiths, R.B., Kaufman, M.: Spin systems on hierarchical lattices. Introduction and thermodynamic limit. Phys. Rev. B26, 5022 (1982) 
5. McKay, S., Berker, A.N., Kirkpatrick, S.: Spin-glass behavior in frustrated Ising models with chaotic renormalization-group trajectories. Phys. Rev. Lett. 48, 767 (1982)

6. Svrakic, N.M., Kertész, J., Selke, W.: Hierarchical lattice with competing interactions and an example of a nonlinear map. J. Phys. A15, L427 (1982)

7. Derrida, B., Eckmann, J.-P., Erzan, A.: J. Phys. A16, 893 (1983)

8. Melrose, J.R.: J. Phys. A16, 1041 (1983); Melrose, J.R.: J. Phys. A16, 3077 (1983)

9. Erzan, A.: Hierarchical $q$-state Potts models with periodic and aperiodic renormalization group trajectories. Phys. Lett. A 93A, 237 (1983)

10. Derrida, B., De Seze, L., Itzykson, C.: J. Stat. Phys, 33, 559 (1983)

11. Julia, G.: J. Math. Pures Appl. 4, 47 (1918)

12. Fatou, P.: Bull. Math. France 47, 161 (1919)

13. Mandelbrot, B.: Ann. N.Y. Acad. Sci. 357, 249 (1980)

14. Douady, A.: Séminaire Bourbaki (Paris) No. 599 (1982-1983)

15. Bessis, D., Geronimo, J., Moussa, P.: J. Stat. Phys. 34, 75 (1984)

16. Widom, M., Bensimon, D., Kadanof, L.P., Shenker, S.J.: Strange objects in the complex plane. J. Stat. Phys. 32, 443 (1983)

17. Itzykson, C., Pearson, R.B., Zuber, J.B.: Nucl. Phys. B220, 415 (1983)

18. Niemeyer, T., Van Leeuwen, J.M.J.: In: Phase transitions and critical phenomena, Vol. 6, Domb, Green (eds.). New York: Academic Press 1976

19. Uzelac, K., Pfeuty, P., Jullien, R. Yang-Lee edge singularity from a real-space renormalization-group method. Phys. Rev. Lett. 43, 805 (1979)

20. Bernasconi, J., Schneider, W.R.: Diffusion on a one-dimensional lattice with random asymmetric transition rates. J. Phys. A15, L729 (1982)

21. Derrida, B., Hilhorst, H.J.: J. Phys. A16, 2641 (1983)

22. Itzykson, C., Luck, J.M.: Proc. of the Brasov Int. School 1983 "Critical phenomena, theoretical aspects"

Communicated by J. Lascoux

Received November 25, 1983

Note added in proof. The equality $\theta_{1}=-\theta_{2}$ can be proven by a duality argument which will be published in another work [22]. 